\section{PCRS-UK News}

Primary Care Respiratory Society UK National Primary Care Respiratory Conference

Inspiring excellence locally: navigating the path to quality respiratory care

11th-12th October 2013, Telford International Centre

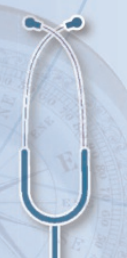

The premier respiratory conference for primary care - offering a route map to quality respiratory care and essential clinical updates for all members of the primary care respiratory team

Respiratory conditions constitute the commonest reason for GP consultations, and the cost of hospitalisations and unscheduled care means that respiratory disease is a high priority for most CCGs and Health Boards. This conference will provide you with the knowledge, examples and skills to help your team navigate the path to quality respiratory care and inspire excellence locally:

- Patient-centred, essential clinical updates from leading experts helping you to provide the right care for your patients, in the right place, at the right time

- 'How to' sessions to build your skills, and help you inspire excellence in respiratory care at practice, locality and regional level

- Cutting edge research sessions run in conjunction with the Primary Care Respiratory Journal - visit www.pcrs-uk.org/abstracts for information on how to submit your abstract

- Presentations and posters with a focus on sharing and implementing best practice examples to meet QIPP targets.

- Network, learn from the experts, and understand the latest development.

- Renowned for its friendly and informal atmosphere, the PCRS-UK conference is a great opportunity to hear from and meet the leading experts in the field, network with like-minded colleagues, and share best practice.

\section{Excellent value for money}

Registration fees start from just $\mathrm{f} 147$ for nurses and $\mathrm{f} 185.85$ for GPs who are PCRS-UK premium members*, inclusive of the Conference dinner and lunches.

If you are not already a paying member* of the PCRS-UK, conference registration includes free premier membership of the PCRS-UK, from the date of registration through to end-2013.

\section{Register NOW to confirm your place}

Visit the conference website NOW for more information and to register at www.pcrs-uk.org/pcrs-uk-annual-conference

* e-connect and practice members can save money on conference registration simply by upgrading to premier membership

PCRS-UK is grateful to the following sponsors of the 2013 conference: Allen \& Hanburys (the respiratory division of GlaxoSmithKline), Almirall Ltd, Boehringer Ingelheim Limited, Napp Pharmaceuticals, Novartis UK and Orion Pharma (UK) Limited.

\section{Respiratory Leaders Skills Workshop June 7th \& 8th June 2013}

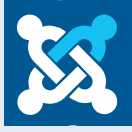

The PCRS-UK Respiratory Leader's Team is delighted to announce that the next workshop will be held on Friday 7th and Saturday 8th June 2013 at Aston Conference Centre Birmingham.

This event will focus on how to build or expand a local respiratory network group in your area. Developing and maintaining a network that drives improvements and in turn supports the patient journey within a joined up pathway is an achievable goal whatever your level of leadership.

We will work with you to:

- Understand where you are currently - Are you within a change resistant hierarchy?

- Decide where you want to get to

- Learn the principles of why networks enable change and improvement

- Realise who to work with and how to engage them

Please sign up to register your interest for this event by emailing mel@pcrs-uk.org or calling 0121767 1928. Places are limited to UK delegates only. Visit the PCRS-UK website for more information http://www.pcrs-uk.org/respiratory-leaders-events

\section{Becoming a member of PCRS-UK}

Whether you're a primary care nurse searching for tried and tested protocols, a GP looking for guidance on COPD

diagnosis and management, or a respiratory specialist interested in

the latest research, it pays to become a member of the Primary Care Respiratory Society UK. And with our new contemporary website offering new and improved functionality later in the year there's never been a better time to join

As a member you'll have unlimited access to a wealth of specialist respiratory care information including newly published resources such as our new opinion sheets on tailoring inhaler choice and cost-effective prescribing.

\section{Join today and get the support of the UK's leading respiratory-care specialists}

\section{Membership packages available from as little as $\mathbf{f 2 7 . 0 0}$ per year}

\section{Great reasons to join PCRS-UK today}

- Make life easier. Become a PCRS-UK member and discover a wealth of credible respiratory care information and resources all in one place

- Keep pace with developments. From COPD guideline changes to managing asthma, there's a best practice resource waiting for you

- Get help with professional development. Save time. It's ready to download and share with your practice staff

- Benefit from expert advice. From managing allergic rhinitis to using spirometry, you'll find a range of concise, easy-to follow Opinion Sheets available free exclusively to members of the society via our website 
- Hard copy of the Primary Care Respiratory Journal. As the flagship publication of the PCRS-UK, this international academic journal provides all the latest primary care respiratory research and top class editorial comment, discussion and educational articles

- Read regular e-alerts. Get the latest respiratory information, news and updates, sent direct to your inbox.

\section{IPCRG News}

\section{IPCRG working locally, collaborating globally}

\section{3rd Scientific Meeting Uppsala 23-24 May 2013}

Registration and abstract submission is open. See http://www.theipcrg.org/display/EVECONF/3rd+Scientific+Meeting\%2C+ Uppsala\%2C+Sweden+23-24+May+2013 for a guide to abstracts, and registration information.

At the opening session we will show excerpts from the brand new TV documentary on FRESH AIR Survey Uganda. The main programme comprises primary care respiratory research: we look forward to your submission.

\section{Abstract deadline: 10 March 2013}

\section{IPCRG 7th World Conference Athens}

The theme and date of the conference are now confirmed:

\section{A Breath of Fresh Air: Multiple Morbidities and Integration 21st - 24th May 2014}

An International Advisory Panel has been invited to ensure we represent different primary care perspectives from around the world. If you wish to be involved, please email Sam Louw at BusinessManager@theipcrg.org

\section{IPCRG Strategy}

The IPCRG Board of directors has recently agreed that over the next three years the IPCRG's priorities are to:

- Promote good clinical respiratory practice

- Actively lead the respiratory health research and education agenda

- Identify and unlock the global potential of our flagship products

- Strengthen our core: membership and communities of interest

\section{Promote good clinical respiratory practice}

We will advocate for primary care's role in lung health by showing practical solutions to case-finding and management that include not only general practice but also other primary care approaches. We will also demonstrate the value of primary care in managing multiple morbidities by helping people change behaviours to stop smoking and/or inhaling indoor smoke, to eat a better diet and to increase physical activity as well as offering safe and effective pharmacological interventions. We will advocate for access for populations to effective respiratory medicines in primary care as part of the UN Global Action Plan for non-communicable diseases.

\section{Actively lead the respiratory health research and education agenda}

We will make progress to answer, or influence others to answer, our prioritised research needs. We will continue to build research and educational capacity in countries with little or no current primary care respiratory research or educational activity including low and middle income countries. We will actively link education and research. We will selectively scale up national projects into larger studies through crossborder collaboration.

\section{Identify and unlock the global potential of our flagship products}

We will expand these flagship products into new clinical and geographical areas:

- IPCRG conferences and respiratory workshops at WONCA conferences

- E-Faculty that increases primary care research capability in respiratory research-naïve countries. In 2013 we will focus on Chile, and also test the model of an IPCRG Research Fellow, where the IPCRG supports the living costs of a researcher who works on relevant primary care questions, to spend time as part of their PhD programme away from their host university

- E-quality - that tests what educational interventions make a difference to clinical behaviour. There are three live projects:

- Asthma education for GPs who prescribe no inhaled medicine in Pune, India

- Introduction to chronic lung disease for physicians in Eritrea

o Improvement of asthma and COPD diagnostic skills in Sao Paulo, Brazil

- FRESH AIR - "franchising" the IPCRG survey by providing IPCRG setup and mentoring support and building a FRESH AIR community by providing mentoring, the FRESH AIR protocol and also some re-usable equipment. This will enable new teams in new countries to measure a population's exposure to smoke, the health impact of that exposure, and to use the survey to develop local commitment and human resources to tackle the problems that are identified.

- UNLOCK - expanding IPCRG's research capacity in COPD and also extending to asthma through cross-sectional and longitudinal studies using our community's data: the UNLOCK group provides access to over 22,000 primary care COPD records and potentially 115,000 primary care asthma records.

- Publications in multiple languages including desktop helpers, position papers, Respiratory@Glance and translations of key Primary Care Respiratory Journal papers

Strengthen our core: membership and communities of interest

We will expand our reach beyond the 100,000 clinicians we reach now through supporting membership drives to new primary care groups, corporate members and associates. The Board and Committee Chairs will actively seek new talent for committee and Board positions. We will strengthen the web platform as the hub for communities of interest and practice

Note: there is currently a call to Members for nominations for President and the host of the 2016 conference. 


\section{ERS General Practice and Primary Care Group 1.6 news}

\section{ERS Congress, Barcelona, 7th - 11th September 2013}

\section{Primary Care Day}

There will again be a Primary Care Day on Saturday morning 7th September. This year the topic is 'Asthma management in clinical practice' and the programme will include a practical overview of telemonitoring, monitoring and achieving asthma control, and selfmanagement. If you are planning to be at the ERS, make sure you arrive in Barcelona in time to join us at what promises to be an excellent session.

\section{Abstract submission}

Last data for submissions was 21st February, but there is always an extension for the ERS and the possibility of submitting 'late breaker' abstracts. So if you haven't already done so, submit your abstract NOW!

\section{PCRJ Award for the best quality abstract submitted to}

\section{Group 1.6}

Worth $€ 500$, and kindly funded by the Primary Care Respiratory Journal, the PCRJ Award will once again be awarded to the best primary care abstract submitted to Group 1.6 for the 2013 ERS Congress in Barcelona. The award is particularly aimed at encouraging young researchers to submit their work
Last year, there were over 100 primary care abstracts selected for the 2012 ERS Congress in Vienna. The top 7 were shortlisted for the PCRJ Award and 3 were highly commended.

The winner of the 2012 ERS Group 1.6 PCRJ Award was:

\section{Abstract Number: 1434}

Title: More than $50 \%$ of patients visit a general practitioner (GP), general physician (Gen $P$ ) or a pediatrician $(P)$ in India for respiratory symptoms: Results of a one-day point-prevalence study in 204,912 patients across 22 states and 5 union territories in India Komalkirti Apte, Monica Barnes, Sapna Madas, Jaideep Gogtay, Sushmeeta Chhowala, Nadar Mahajan and Sundeep Salvi. Chest Research Foundation, Pune, Maharashtra, India; CIPLA LTD, Mumbai, Maharashtra, India.

\section{Joining the ERS}

Are you an ERS member? If you're not and you regularly attend the ERS Congress, why not join the ERS and receive all the membership benefits? If you are thinking of joining, please ensure that you join Group 1.6, the General Practice and Primary Care Group.

Bjorn Stallberg, Chair Group 1.6 Hilary Pinnock, Secretary Group 1.6

\section{Available online at http://www.thepcrj.org}
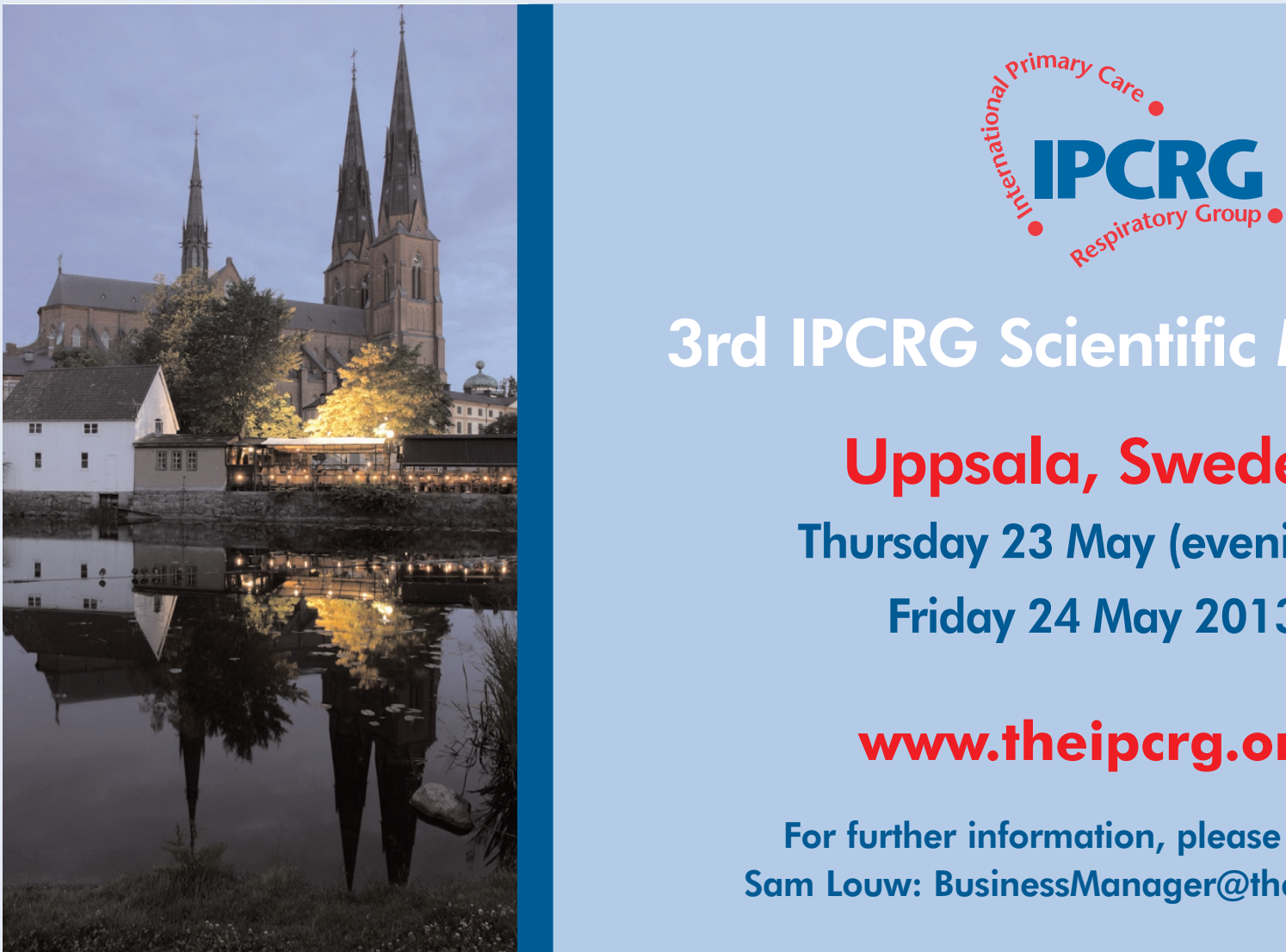

\section{3rd IPCRG Scientific Meeting \\ Uppsala, Sweden}

Thursday 23 May (evening) Friday 24 May 2013

\section{www.theipcrg.org}

For further information, please contact Sam Louw: BusinessManager@theipcrg.org 